\title{
Improvements on the Duality Based Method Used in Solving Optimal Power Flow Problems
}

\author{
Ch'i-Hsin Lin, Shin-Yeu Lin, and Shieh-Shing Lin
}

\begin{abstract}
To improve the previously developed dual-type (DT) method used in solving optimal power flow (OPF) problems with large number of thermal-limit constraints, we propose two new techniques in this paper. The first one is a graph-method based decomposition technique which can decompose the large-dimension projection problem, caused by the large number of thermal-limit constraints, into several independent medium-dimension projection subproblems at the expense of slight increment of the dual problem's dimension. The second technique is an active-set strategy based DT method which can solve the medium-dimension projection subproblems efficiently. We have used the DT method embedded with these two new techniques in solving numerous OPF's with large number of thermal-limit constraints. The test results show that the proposed techniques are very efficient and effectively improve the DT method for handling large number of thermal-limit constraints.
\end{abstract}

Index Terms-Decomposition, graph method, nonlinear programming, optimal power flow (OPF), thermal-limit constraints.

\section{INTRODUCTION}

$\mathbf{L}$ ARGE-SCALE optimal power flow (OPF) problem has been a typical research issue in the power system research history. Among all existing algorithms developed for solving this problem such as [1]-[10], efficiency and numerical stability are two major goals to pursue. In previous research work [11], we have developed a dual-type (DT) method to solve the quadratic programming problems induced from using a successive quadratic programming (SQP) method to solve OPF problems. This DT method is numerically stable and circumvent the difficulties of binding inequality constraints; most of its computations lie in two steps: the first one is solving a set of linear equations to obtain an ascent direction of the dual function, and the other one is solving a large-dimension projection problem so as to compute the gradient of the dual function required in setting up the above mentioned linear equations. It has been shown in [11] that this DT method is computationally very efficient provided that the target systems possess a) sparsity structure so that we can use a sparse-matrix technique to solve the set of linear equations and b) decoupling inequality constraints so that we can decompose the large-dimension projection problem into independent branchwise or nodewise projection subproblems.

Manuscript received September 24, 1999; revised September 28, 2001. This work was supported in part by the National Science Council of Taiwan, R.O.C under Grant NSC88-2213-E-009-123.

C.-H. Lin is with the Department of Electronics Engineering, KaoYuan Institute of Technology, Kaoshiung, Taiwan, R.O.C.

S.-Y. Lin and S.-S. Lin are with the Department of Electrical and Control Engineering, National Chiao Tung University, Hsinchu, Taiwan, R.O.C., (e-mail: sylin@cc.nctu.edu.tw).

Publisher Item Identifier S 0885-8950(02)03837-3.
The OPF problems with voltage and generation constraints possess the above two properties. However, if the thermal-limit constraints are considered, property b) may no longer be valid when these transmission lines form a connecting graph. This implies that the large-dimension projection problem induced in the DT method cannot be decomposed into branchwise and nodewise projection subproblems, hence, increase computational inefficiency. A remedy strategy proposed in [11] is to convert the coupling thermal-limit constraints into equality constraints and decoupling branchwise inequality constraints using slack variables. Such a conversion will make property b) hold, however, at the expense of increasing the number of equality constraints, slack variables, and consequently the dimension of the dual problem, which causes computational inefficiency. Thus, we encounter two extreme cases corresponding to whether using the conversion technique or not. In fact, compromising between these two extreme cases to generalize the DT method to deal with the quadratic subproblems of the OPF problems with large number of thermal-limit constraints is the purpose of this paper.

To accomplish this goal, we propose two techniques. The first one is a graph method based decomposition technique which incorporates with the conversion technique can decompose the coupling thermal-limit constraints into disjoint subsets so that the large-dimension projection problem induced in the DT method can be decomposed into independent medium-dimension projection subproblems. The second technique is an active-set strategy based DT method which can solve the medium-dimension projection subproblems efficiently. These two new techniques to be presented in Sections II and III can be embedded in the DT method to incorporate with the SQP method to solve the OPF problems with large number of thermal-limit constraints as presented in Section IV. Test results are given in Section $\mathrm{V}$ to demonstrate the efficiency of the proposed techniques and their effective improvement on the DT method.

\section{GRAPH-Method BASED Decomposition TECHNIQUe}

The thermal-limit constraint for a transmission line in power systems [12] can be expressed as

$$
\underline{h}_{i j} \leq h_{i j}\left(e_{i}, f_{i}, e_{j}, f_{j}\right) \leq \bar{h}_{i j}
$$

where $h_{i j}\left(e_{i}, f_{i}, e_{j}, f_{j}\right)$ denotes the real power flow of the transmission line from bus $i$ to bus $j, e_{i}$ and $f_{i}$ denote real and imaginary parts of bus $i$ 's voltage, respectively, and $\underline{h}_{i j}$ and $\bar{h}_{i j}$ denote the lower and upper limit of the real power flow, respectively. Thus, whenever the thermal-limit constraints are required upon a set of connecting transmission lines, they are coupling. 


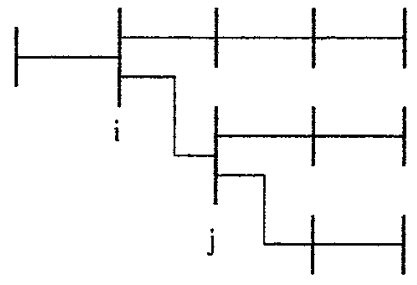

Fig. 1. Example of category a).

\section{A. Converting Thermal-Limit Constraints}

Using slack variable $z_{i j}$, we can convert (1) into the following equality constraint and branchwise inequality constraint [11]:

$$
\begin{aligned}
h_{i j}\left(e_{i}, f_{i}, e_{j}, f_{j}\right) & =z_{i j} \\
\underline{h}_{i j} & \leq z_{i j} \leq \bar{h}_{i j} .
\end{aligned}
$$

\section{B. Decomposition Algorithm}

As indicated in Section I, to render the OPF problem with large number of coupling thermal-limit constraints, we should compromise between increasing the dimension of the dual problem, caused by additional equality constraints, and reducing the dimension of the projection subproblem. To fulfill this task, a natural criteria can be minimizing the increase of equality constraints subject to keeping the dimensions of the projection subproblems under certain level. We present in this section a graph-method based decomposition technique to accomplish the above mentioned criteria approximately.

Construction of the Graph: An advantage of the thermallimit constraints of a power system is these transmission lines themselves form a graph.

Categories of the Graph: There may exist separate connecting graphs corresponding to decoupling subsets of thermal-limit constraints. Thus, our graph-method based decomposition technique should apply to each individual connecting graph. In this section, we denote a connecting graph by $\mathcal{G}$. First of all, we categorize $\mathcal{G}$ into four categories: a) no loop, b) few loops and each loop contains few transmission lines, c) few loops and some loops contain many transmission lines, and d) many loops. To perform the categorization, we first calculate the number of loops $L$ and the number of transmission lines $T_{l_{j}}$ in each loop $l_{j}, j=1, \ldots, L$. If $L=0$, $\mathcal{G}$ is of category a); Fig. 1 shows such an example. We let $L_{0}$ and $T_{0}$ denote the threshold values of $L$ and $T_{l_{j}}$, respectively. If $L<L_{0}$ and $T_{l_{j}}<T_{0}$ for every $j=1, \ldots, L$, then $\mathcal{G}$ is of category b); suppose we set $L_{0}=4, T_{0}=10$; Fig. 2(a) shows such an example. If $L<L_{0}$ and $T_{l_{j}} \geq T_{0}$ for some $j$, then $\mathcal{G}$ is of category c); Fig. 3(a) shows such an example, in which $T_{l_{1}}=11>T_{0}=10$. If $L \geq L_{0}, \mathcal{G}$ is of category d); Fig. 4(a) shows such an example.

Remark 1: To calculate $L$ and $T_{l_{j}}, j=1, \ldots, L$, for $\mathcal{G}$ is indeed a simple task. We can apply any typical spanning tree algorithm [13] to $\mathcal{G}$ to identify a directed spanning tree starting from the root bus, which is called source bus in the sequel. Then each transmission line not in this tree will form a loop with transmission lines in this tree. Subsequently, we may calculate $L$ and $T_{l_{j}}, j=1, \ldots, L$.

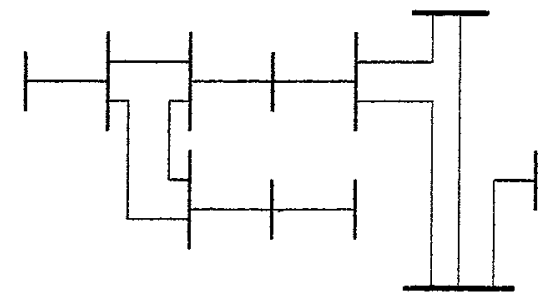

(a)

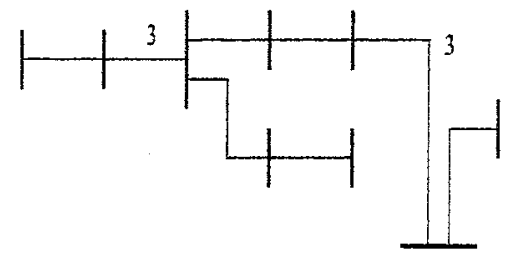

(b)

Fig. 2. (a) Example of category b). (b) Modification of Fig. 2(a).

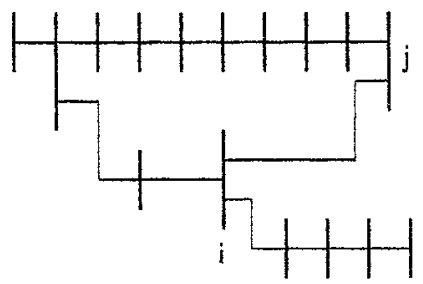

(a)

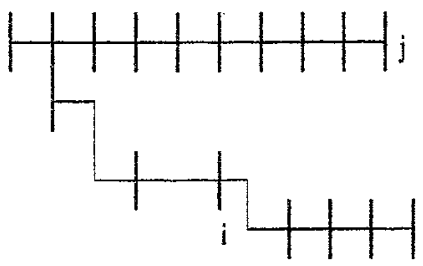

(b)

Fig. 3. (a) Example of category c). (b) Modification of Fig. 3(a).

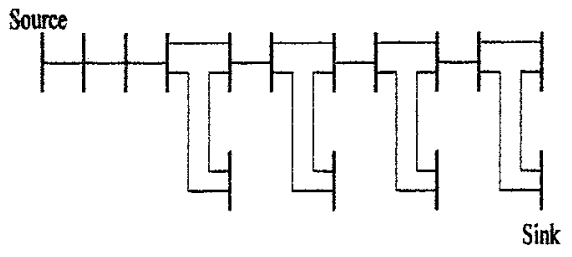

(a)

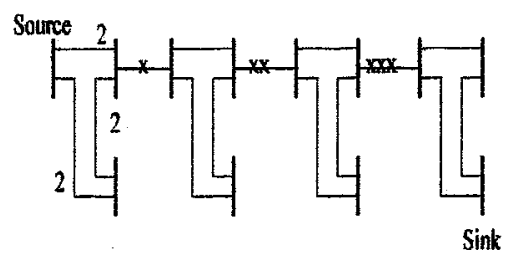

(b)

Fig. 4. (a) Example of category d). (b) Modification of Fig. 4(a).

\section{Graph-Method Based Decomposition Algorithm}

The criteria that we mention at the beginning of Section II-B can be interpreted in terms of the represented graph as: to determine a set of smallest possible number of transmission lines so 
that the removal of which ensures the dimensions of the separated subgraphs under certain level; in other words, to make the dimension of the largest separated subgraph as small as possible. Our decomposition procedures for the graph of each category is heuristic and aims to accomplish this criteria approximately.

Before proceeding, we need the following notations: $t_{i j}$ denotes the transmission line connecting bus $i$ with bus $j ; T_{r}$ denotes a set of transmission lines in $\mathcal{G} ; S_{j}$ denotes the $j$ th subgraph of $\mathcal{G} ; S_{j}\left(T_{r}\right)$ denotes the $j$ th subgraph of $\mathcal{G}$ after the removal of $T_{r}$. In addition, we need a terminology capacity denoted by $C$ so that $C(*)$ represents the total number of transmission lines in $*$; for example, $C\left(t_{i j}\right)=1$. To ensure the dimension of a subgraph being under certain level, we define a threshold value $C_{0}$ so that if $C\left(S_{j}\left(T_{r}\right)\right)>C_{0}, S_{j}\left(T_{r}\right)$ need be decomposed further. Using these notations, we can state our decomposition algorithm in the following and provide illustrative examples for those steps that perform decomposition afterwards.

\section{Algorithm I:}

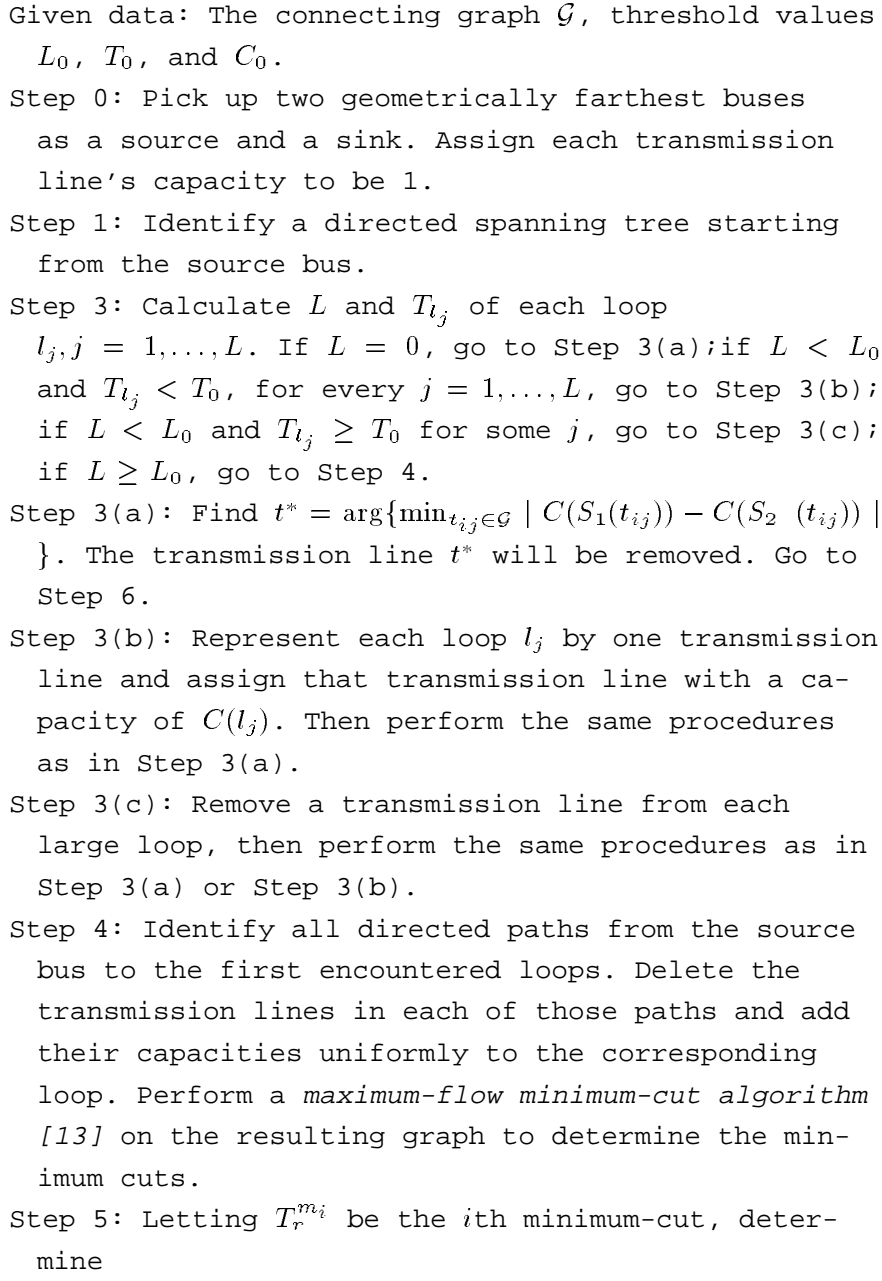

$$
T_{r}^{m^{*}}=\arg \left\{\min _{T_{r}^{m_{i}}}\left[\max _{S_{j}\left(T_{r}^{m_{i}}\right)} C\left(S_{j}\left(T_{r}^{m_{i}}\right)\right)\right]\right\} .
$$

Then $T_{r}^{m^{*}}$ will be removed.

Step 6: For any separated subgraph $S_{j}$, if $C\left(S_{j}\right) \leq C_{0}$, pass $S_{j}$; otherwise, repeat Steps 1 to 6 for $S_{j}$.
Note: A minimum cut is a cutset with minimum total capacities among all the cutsets that can separate the graph into at least two disjoint subgraphs, and the source bus and sink bus belong to different subgraphs.

\section{Illustrations and Examples for Decomposition Steps of Algorithm I}

In the above algorithm, the removed transmission line(s) implies the corresponding thermal-limit constraints will be converted to equality and branchwise inequality constraints as shown in (2) and (3). For the graphical examples provided here, the capacity of a transmission line is 1 unless it is specifically marked. Following are the illustrations for the decomposition steps:

For Step 3(a): The removal of a transmission line in $\mathcal{G}$ of category (a) will result in two separated subgraphs $S_{1}$ and $S_{2}$. For example, in Fig. 1, $\left|C\left(S_{1}\left(t_{i j}\right)\right)-C\left(S_{2}\left(t_{i j}\right)\right)\right|=0$. Hence $t_{i j}$ will be removed. This procedure achieves the destined criteria.

For Step 3(b): Fig. 2(b) is resulted from Fig. 2(a) using one transmission line to represent a loop $l_{j}$ associated with the capacities $C\left(l_{j}\right)$. Consequently, the resulting graph in Fig. 2(b) is of category (a).

For Step 3(c): Fig. 3(b) belonging to category a) is resulted from Fig. 3(a) after removing $t_{i j}$.

For Step 4: The reason to delete those transmission lines indicated in Step 4 is to avoid meaningless minimum-cut. For example, the transmission line connecting with the source bus in Fig. 4(a) is an obvious minimum cut to separate the source bus from the sink bus. Thus, Fig. 4(b) is resulted from Fig. 4(a) after deleting the transmission lines from the source bus to the first encountered loop, and the capacities of the transmission lines of that loop are also modified as dictated in Step 4.

For Step 5: Equation (4) is to find the minimum cut such that the dimension of the largest separated subgraph due to the removal of that cut is smallest. For example, in Fig. 4(b), each of the transmission lines marked by " $x$," "x $\mathrm{x}$," and " $\mathrm{x} x \mathrm{x}$," represents a minimum cut, and we denote them by $T_{r}^{\times}, T_{r}^{\times \times}$, and $T_{r}^{\times \times \times}$, respectively. Consequently, $\max _{S_{j}\left(T_{r}^{\times}\right)} C\left(S_{j}\left(T_{r}^{\times}\right)\right)=11, \max _{S_{j}\left(T_{r}^{\times \times}\right)} C\left(S_{j}\left(T_{r}^{\times \times}\right)\right)=$ 10 , and $\max _{S_{j}\left(T_{r}^{\times \times \times}\right)} C\left(S_{j}\left(T_{r}^{\times \times \times}\right)\right)=14$. Therefore, $T_{r}^{\times \times}$ will be the minimum cut to be removed.

Remark 2: Algorithm I can be executed off-line, because the thermal-limit constraints required upon the transmission lines are usually known in prior.

Remark 3: The factors that really affect the computational speed of our method proposed in this paper is the reduction of the projection subproblems' dimensions, in terms of the capacities of the disjoint subgraphs, and the additional equality constraints and slack variables, in terms of the removed branches, however, not the category of the graph. The purpose that we categorize the graph into four categories is to find a systematic method, as shown in Steps 3(a)-(c), 4 and 5 of Algorithm I, to decouple the connecting graph into disjoint subgraphs by removing as less branches as possible so as to achieve the goal of our decomposition strategy approximately.

Remark 4: Since this graph-method based decomposition algorithm is executed off-line, the values of $L_{0}$ and $T_{0}$ can be determined based on the structure of $\mathcal{G}$ In general, for a larger 
$C(\mathcal{G})$, there may be more loops, and each loop may consist of more transmission lines. Thus, the values of $L_{0}$ and $T_{0}$ should be set larger for larger $C(\mathcal{G})$. Recommended value ranges of $L_{0}$ and $T_{0}$ are $(4,8)$ and $(10,15)$, respectively, depending on the value of $C(\mathcal{G})$. Comments regarding the value of $C_{0}$ will be presented in Remark 5.

\section{METHOD FOR MEDIUM-DIMENSION PROJECTION SUBPROBLEM}

The following projection subproblem is considered in this section:

$$
\begin{aligned}
& \min _{y}(y-\tilde{y})^{T}(y-\tilde{y}) \\
& \underline{c}_{i} \leq a_{i}^{T} y+b_{i} \leq \bar{c}_{i}, i=1, \ldots, n \\
& y \in \Omega
\end{aligned}
$$

where the $n$-dimensional vector $\tilde{y}$ is a given vector consisting of the increments of the real part and imaginary part of the bus voltage during solution process, $c_{i}, \bar{c}_{i}$, and $b_{i}$ are scalars, $a_{i}$ is an $n$-dimensional constant vector, and the functional inequality constraints (6) denote the linearized coupling thermal-limit constraints, $\Omega$ denotes a set of nodewise inequality constraints such as the voltage-magnitude constraints and control-variable constraints.

The method we propose to solve (5)-(7) is an active-set strategy based DT method. The active-set strategy will first identify the working set, that is the active linearized thermal-limit constraints at current state $y_{k-1}$; then at the next, $k$-th, iteration, we will solve the problem (5)-(7) for the working set at $y_{k-1}$ only as shown below

$$
\begin{aligned}
\min _{y}(y-\tilde{y})^{T}(y-\tilde{y}) \\
a_{i}^{T} y+b_{i}=d_{i}, i \in J\left(y_{k-1}\right) \\
y \in \Omega
\end{aligned}
$$

where $J\left(y_{k-1}\right)$ denotes the set of indices of active linearized thermal-limit constraints (6) at $y_{k-1}$, that is $J\left(y_{k-1}\right)=\{i \in$ $(1,2, \ldots, n) \mid \underline{c}_{i} \geq a_{i}^{T} y_{k-1}+b_{i}$ or $\left.\bar{c}_{i} \leq a_{i}^{T} y_{k-1}+b_{i}\right\}, d_{i}$ denotes either $\underline{c}_{i}$ or $\bar{c}_{i}$ depending on which side of the linearized thermal-limit constraint in (6) is active. The initial working set is assigned to be $J\left(y_{0}\right)=J(\tilde{y})$. Clearly, the working set $J\left(y_{k-1}\right)$ should be updated for each newly obtained $y_{k}$, which is the solution of (8), and the details will be discussed later.

To solve (8), we will solve its corresponding dual problem

$$
\max _{\mu} q(\mu)
$$

where the dual function

$$
q(\mu)=\min _{y \in \Omega}(y-\tilde{y})^{T}(y-\tilde{y})+\sum_{i \in J\left(y_{k-1}\right)} \mu_{i}\left(a_{i}^{T} y+b_{i}-d_{i}\right)
$$

and $\left[\mu_{i}, i \in J\left(y_{k-1}\right)\right]$ is the Lagrange multiplier vector associated with the active constraints. The dual problem (9) can be solved by the DT method proposed in [11] using the following iterations:

$$
\mu(j+1)=\mu(j)+\beta(j) \Delta \mu(j)
$$

where $\beta(j)$ is a positive step-size and $\Delta \mu(j)$ can be obtained from solving

$$
Q(\mu(j)) \Delta \mu(j)+\frac{\partial q(\mu(j))}{\partial \mu}=0
$$

in which the matrix $Q(\mu(j))$ is an approximate Hessian of $q(\mu(j))$, which can be computed by the following [14]:

$$
Q(\mu(j))=-A_{y_{k-1}}^{T} A_{y_{k-1}}-\delta_{1} I
$$

where the matrix $A_{y_{k-1}}=\left[a_{i}\right], i \in J\left(y_{k-1}\right)$ is the collection of the column vectors $a_{i}, i \in J\left(y_{k-1}\right), \delta_{1}$ is a small positive real number, and $I$ is an identity matrix. Each component of the gradient of $q(\mu(j))$ in (12) can be computed by [14]

$$
\frac{\partial q(\mu(j))}{\partial \mu_{i}}=a_{i}^{T} \hat{y}^{\prime}+b_{i}-d_{i}, i \in J\left(y_{k-1}\right)
$$

where $\hat{y}^{\prime}$, according to the DT method in [11], is the solution of the minimization problem on the RHS of (10), which is equivalent to the following decomposable projection problem

$$
\min _{y^{\prime} \in \Omega}\left(y^{\prime}-\tilde{y}^{\prime}\right)^{T}\left(y^{\prime}-\tilde{y}^{\prime}\right)
$$

where

$$
\tilde{y}^{\prime}=\tilde{y}-\frac{1}{2} \sum_{i \in J\left(y_{k-1}\right)} \mu_{i} a_{i} .
$$

The projection problem (15) can be decomposed into independent nodewise projection subproblems, and the analytical formula for solving each nodewise projection subproblem is given in [11]. Therefore, setting up the linear equation (12) is an easy task.

Consequently, if there are only few active linearized thermallimit constraints in each iteration, which is typical in normal operating conditions of a power system [12], then the dimension of the linear equation (12) as well as $-A_{y_{k-1}}^{T} A_{y_{k-1}}-\delta I$ should be small, and (12) can be easily solved. Therefore, the advantages of the DT method are preserved in this solution process.

Addition and Subtraction for the Constraints in the Working Set: When $y_{k}$, the solution of (8), is obtained, we will check its satisfaction of (6). Any new active index will be added to $J\left(y_{k-1}\right)$ to update $J\left(y_{k}\right)$. On the contrary, if no constraint in (6) is violated, we will investigate the following condition: if $d_{i}=\bar{c}_{i}$ and $\mu_{i}<0$ or $d_{i}=\underline{c}_{i}$ and $\mu_{i}>0$, for some $i \epsilon$ $J\left(y_{k-1}\right)$, then this constraint will be released from $J\left(y_{k-1}\right)$ to update $J\left(y_{k}\right)[14]$. Consequently, if no constraint is violated, and if $d_{i}=\bar{c}_{i}$ and $\mu_{i} \geq 0$ or $d_{i}=\underline{c}_{i}$ and $\mu_{i} \leq 0$, for all $i \in J\left(y_{k-1}\right)$, then $y_{k}$ is the solution of the projection subproblem (5)-(7).

In the following, we state the algorithm of active-set strategy based DT method for solving the projection subproblem (5)-(7).

Algorithm II:

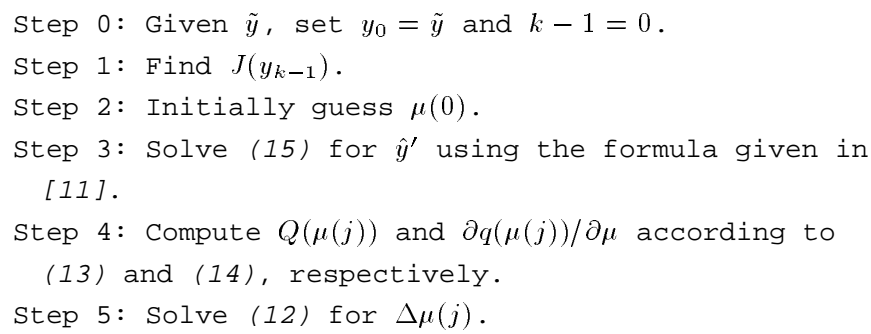




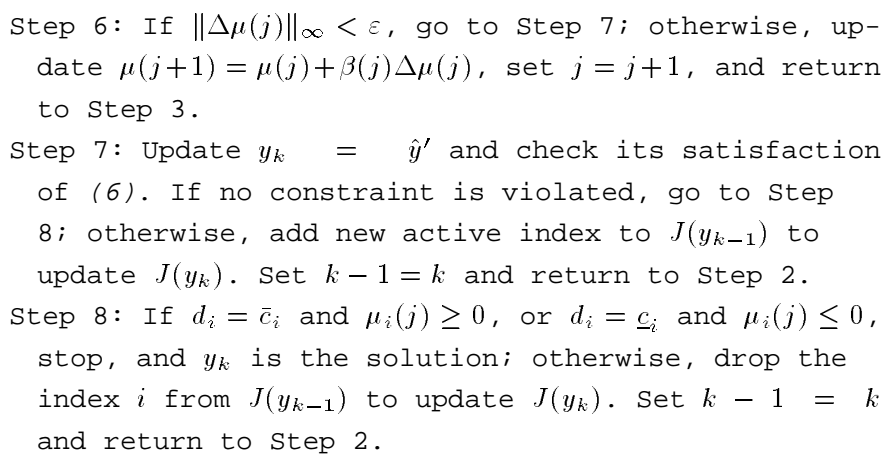

Remark 5: As indicated previously, our active set strategy based DT method is especially suitable for solving projection problem with very few active constraints. However, if more active constraints are present, smaller $C_{0}$ in Algorithm I is preferable so as to avoid the frequent adding and dropping the active constraints during the solution process. Thus, the choice of the value of $C_{0}$ in Algorithm I will depend on the value of $C(\mathcal{G})$ and the system's statistics. In general, the value range of $C_{0}$ is taken to be $(15,40)$.

\section{METHOD FOR OPF PROBLEM With LARGE THERMAL-LIMIT CONSTRAINTS}

Using notations given in [11], we can state the OPF problem with large number of thermal-limit constraints as follows:

$$
\begin{gathered}
\min _{x} F(x) \\
g(x)=0 \\
\underline{h} \leq h(e, f) \leq \bar{h} \\
\underline{V} \leq V \leq \bar{V} \\
\underline{u} \leq u \leq \bar{u}
\end{gathered}
$$

where the vector $x=(u, e, f)$ represents all variables, in which the vector $u$ represents control variables such as $P_{G}, Q_{G}$, transformer tap ratio, etc.; $g(x)=0$ denotes the real and reactive power flow balance equations at all but slack buses; $e$ and $f$ are real and imaginary parts of the bus complex voltage; the vector function $h(e, f)$ denotes the transmission line real power flow functions, the vectors $\underline{h}$ and $\bar{h}$ denote the lower and upper limit of real power line flows, respectively, that is, the thermal-limit; $\underline{V}$ and $\bar{V}$ are lower and upper limit of bus voltage magnitudes, and $\underline{u}$ and $\bar{u}$ are lower and upper limits of control variables.

We will first apply Algorithm I described in Section II to decompose the coupling thermal-limit constraints $\underline{h} \leq h(e, f) \leq$ $\bar{h}$. We let $\underline{h}_{1} \leq h_{1}\left(e_{1}, f_{1}\right) \leq \bar{h}_{1}, \underline{h}_{2} \leq h_{2}\left(e_{2}, f_{2}\right) \leq \bar{h}_{2}, \ldots$, and $\underline{h}_{n} \leq h_{n}\left(e_{n}, f_{n}\right) \leq \bar{h}_{n}$ denote the $n$ disjoint subsets of thermal-limit constraints resulted from Algorithm I, and let $\underline{h}_{0} \leq h_{0}(e, f) \leq \bar{h}_{0}$ denote the set of thermal-limit constraints that will be converted to equality constraints and branchwise inequality constraints. After the decomposition and conversion, the OPF problem (17) becomes

$$
\begin{aligned}
\min _{x} F(x) & \\
g(x) & =0 \\
h_{0}(e, f)-z & =0 \\
\underline{h}_{0} & \leq z \leq \bar{h}_{0} \\
\underline{h}_{i} & \leq h_{i}\left(e_{i}, f_{i}\right) \leq \bar{h}_{i}, i=1,2, \ldots, n \\
\underline{V} & \leq V \leq \bar{V} \\
\underline{u} & \leq u \leq \bar{u}
\end{aligned}
$$

where $z$ is the vector of slack variables used to convert the inequality constraints $\underline{h}_{0} \leq h_{0}(e, f) \leq \bar{h}_{0}$ into equality constraints $h_{0}(e, f)-z=0$ and branchwise inequality constraints $\underline{h}_{0} \leq z \leq \bar{h}_{0}$. The SQP method [11] uses the following iterations to solve the above OPF problem:

$$
\begin{aligned}
& x(k+1)=x(k)+\alpha(k) \Delta x(k) \\
& z(k+1)=z(k)+\alpha(k) \Delta z(k)
\end{aligned}
$$

where $\alpha(k)$ is a step-size [11], and $(\Delta x(k), \Delta z(k))$ is the solution of the following QP subproblem:

$$
\begin{gathered}
\min _{\Delta x, \Delta z} \frac{\partial F(x(k))}{\partial x} \Delta x+\frac{1}{2} \Delta x^{T} H \Delta x+\frac{1}{2} \eta \Delta z^{T} \Delta z \\
g(x(k))+\frac{\partial g(x(k))}{\partial x} \Delta x=0 \\
h_{0}(k)+\frac{\partial h_{0}(k)}{\partial e} \Delta e+\frac{\partial h_{0}(k)}{\partial f} \Delta f-z(k)-\Delta z=0 \\
\underline{h}_{0} \leq z(k)+\Delta z \leq \bar{h}_{0} \\
\underline{h}_{i} \leq h_{i}(k)+\frac{\partial h_{i}(k)}{\partial e_{i}} \Delta e_{i}+\frac{\partial h_{i}(k)}{\partial f_{i}} \Delta f_{i} \leq \bar{h}_{i} \\
\underline{V} \leq V(k)+\frac{\partial V(k)}{\partial e} \Delta e+\frac{\partial V(k)}{\partial f} \Delta f \leq \bar{V} \\
\underline{u} \leq u(k)+\Delta u \leq \bar{u}
\end{gathered}
$$

in which $h_{i}(k)$ denotes $h_{i}\left(e_{i}(k), f_{i}(k)\right)$, and the diagonal matrix $H$ is defined by

$$
H \equiv \operatorname{diag}\left[\frac{\partial^{2} F(x(k))}{\partial x^{2}}\right]+\frac{1}{2} \delta_{2} I
$$

where $I$ is an identity matrix, and $\delta_{2}$ is a small positive real number but enough to make $H$ positive definite.

Remark 6: In most of the OPF problems such as economic dispatch or minimum system-losses problems, $\delta_{2}$ can be predetermined.

The dual problem of the above QP subproblem is

$$
\max _{\lambda} \phi(\lambda)
$$


where the dual function

$$
\begin{aligned}
\phi(\lambda)= & \min _{(\Delta x, \Delta z) \in \Gamma} \frac{\partial F(x(k))}{\partial x} \Delta x+\frac{1}{2} \Delta x^{T} H \Delta x \\
& +\frac{1}{2} \eta \Delta z^{T} \Delta z+\lambda_{g}^{T}\left[g(x(k))+\frac{\partial g(x(k))}{\partial x} \Delta x\right] \\
& +\lambda_{h_{0}}^{T}\left[h_{0}(k)+\frac{\partial h_{0}(k)}{\partial e} \Delta e+\frac{\partial h_{0}(k)}{\partial f} \Delta f\right. \\
& -z(k)-\Delta z]
\end{aligned}
$$

and the constraints of the primal variables in the dual function,

$$
\begin{aligned}
\Gamma= & \left\{(\Delta x, \Delta z) \mid \underline{h}_{i} \leq h_{i}(k)+\frac{\partial h_{i}(k)}{\partial e_{i}} \Delta e_{i}+\frac{\partial h_{i}(k)}{\partial f_{i}} \Delta f_{i} \leq \bar{h}_{i}\right. \\
& i=1, \ldots, n, \underline{h}_{O} \leq z(k)+\Delta z \leq \bar{h}_{o} \\
& \underline{V} \leq V(k)+\frac{\partial V(k)}{\partial e} \Delta e+\frac{\partial V(k)}{\partial f} \Delta f \leq \bar{V} \\
& \underline{u} \leq u(k)+\Delta u \leq \bar{u}\} .
\end{aligned}
$$

The DT method solves the above dual problem using the iterations

$$
\lambda(t+1)=\lambda(t)+\beta(t) \Delta \lambda(t)
$$

where $t$ is the iteration index, $\beta(t)$ is a positive step-size, and $\Delta \lambda(t)=\left(\Delta \lambda_{g}(t), \Delta \lambda_{h_{0}}(t)\right)$ is obtained from solving

$$
\frac{\partial^{2} \phi^{u}(\lambda(t))}{\partial \lambda^{2}} \Delta \lambda(t)+\frac{\partial \phi^{T}(\lambda(t))}{\partial \lambda}=0
$$

in which the Hessian of $\phi^{u}(\lambda(t))$ and the gradient of $\phi(\lambda(t))$ can be computed as described in the following.

The $\phi^{u}(\lambda(t))$ is the unconstrained dual function defined as

$$
\begin{aligned}
\phi^{u}(\lambda(t))= & \min \frac{\partial F(x(k))}{\partial x} \Delta x \\
& +\frac{1}{2} \Delta x^{T} H \Delta x+\frac{1}{2} \eta \Delta z^{T} \Delta z \\
& +\lambda_{g}^{T}\left[g(x(k))+\frac{\partial g(x(k))}{\partial x} \Delta x\right] \\
& +\lambda_{h_{0}}^{T}\left[h_{0}(k)-z(k)+\frac{\partial h_{0}(k)}{\partial e} \Delta e\right. \\
& \left.+\frac{\partial h_{0}(k)}{\partial f} \Delta f-\Delta z\right]
\end{aligned}
$$

which is the dual function in (23) but relaxing the constraints on primal variables, and the Hessian of $\phi^{u}(\lambda(t))$ can be computed by

$$
\begin{aligned}
& \frac{\partial^{2} \phi^{u}(\lambda(t))}{\partial \lambda^{2}}= \\
& -\left[\begin{array}{cc}
\frac{\partial g(x(k))}{\partial x} H^{-1} \frac{\partial g^{T}(x(k))}{\partial x} & \frac{\partial g(x(k))}{\partial x} H^{-1} \frac{\partial h_{0}^{T}(k)}{\partial x} \\
\frac{\partial h_{0}(k)}{\partial x} H^{-1} \frac{\partial g^{T}(x(k))}{\partial x} & \frac{\partial h_{0}(k)}{\partial x} H^{-1} \frac{\partial h_{0}^{T}(k)}{\partial x}+\frac{1}{\eta} I
\end{array}\right] .
\end{aligned}
$$

The gradient of $\phi(\lambda(t))$ is

$$
\frac{\partial \phi^{T}(\lambda(t))}{\partial \lambda}=\left(\frac{\partial \phi(\lambda(t))}{\partial \lambda_{g}}, \frac{\partial \phi(\lambda(t))}{\partial \lambda_{h_{0}}}\right)^{T}
$$

and can be computed by

$$
\begin{aligned}
\frac{\partial \phi^{T}(\lambda(t))}{\partial \lambda_{g}}= & g(x(k))+\frac{\partial g(x(k))}{\partial x} \Delta \hat{x} \\
\frac{\partial \phi^{T}(\lambda(t))}{\partial \lambda_{h_{0}}}= & h_{0}(k)-z(k)+\frac{\partial h_{0}(k)}{\partial e} \Delta \hat{e} \\
& +\frac{\partial h_{0}(k)}{\partial f} \Delta \hat{f}-\Delta \hat{z},
\end{aligned}
$$

where $(\Delta \hat{x}, \Delta \hat{z})$ needed in (29) and (30) is the solution of the constrained minimization problem on the RHS of (23) and, according to the DT method [11], can be obtained from solving the following projection problem:

$\min _{(\Delta x, \Delta z) \in \Gamma}(\Delta z-\Delta \tilde{z})^{T}(\Delta z-\Delta \tilde{z})+(\Delta x-\Delta \tilde{x})^{T}(\Delta x-\Delta \tilde{x})$

where $(\Delta \tilde{z}, \Delta \tilde{x})$ computed by

$$
\begin{aligned}
\Delta \tilde{z}= & \frac{\lambda_{h_{0}}}{\eta} \\
\Delta \tilde{x}= & -H^{-1}\left[\frac{\partial F^{T}(x(k))}{\partial x}+\frac{\partial g^{T}(x(k))}{\partial x} \lambda_{g}\right. \\
& \left.+\frac{\partial h_{0}^{T}(k)}{\partial x} \lambda_{h_{0}}\right]
\end{aligned}
$$

is the solution of the unconstrained dual function $\phi^{u}(\lambda(t))$ shown in (26). Furthermore, $\Gamma$ can be expressed as

$$
\Gamma=\Gamma_{z} \cup\left(\cup_{i=1}^{n} \Gamma_{x_{i}}\right)
$$

where $\Gamma_{z}=\left\{\Delta z \mid \underline{h}_{o} \leq z(k)+\Delta z(k) \leq \bar{h}_{o}\right\}$ and

$$
\begin{aligned}
\Gamma_{x_{i}}= & \left\{\Delta x_{i} \mid \underline{h}_{i} \leq h_{i}(k)+\frac{\partial h_{i}(k)}{\partial e_{i}} \Delta e_{i}+\frac{\partial h_{i}(k)}{\partial f_{i}} \Delta f_{i} \leq \bar{h}_{i}\right. \\
& \underline{V}_{i} \leq V_{i}(k)+\frac{\partial V_{i}(k)}{\partial e_{i}} \Delta e_{i}+\frac{\partial V_{i}(k)}{\partial f_{i}} \Delta f_{i} \leq \bar{V}_{i} \\
& \left.\underline{u}_{i} \leq u_{i}(k)+\Delta u_{i} \leq \bar{u}_{i}\right\}, i=1, \ldots, n .
\end{aligned}
$$

Since $\Gamma_{z}$ and $\Gamma_{x_{i}}, i=1, \ldots, n$, are $n+1$ disjoint subsets, the projection problem (31) can be decomposed into the following $n+1$ projection subproblems:

$$
\min _{\Delta z \in \Gamma_{z}}(\Delta z-\Delta \tilde{z})^{T}(\Delta z-\Delta \tilde{z})
$$

and

$$
\min _{\Delta x_{i} \in \Gamma_{x_{i}}}\left(\Delta x_{i}-\Delta \tilde{x}_{i}\right)^{T}\left(\Delta x_{i}-\Delta \tilde{x}_{i}\right), i=1, \ldots, n
$$


where $\Delta \tilde{z}$ and $\left(\Delta \tilde{x}_{1}, \Delta \tilde{x}_{2}, \ldots, \Delta \tilde{x}_{n}\right)=\Delta \tilde{x}$ are given in (32) and (33), respectively. The projection subproblem in (35) can be easily solved by the following analytical formula:

$$
\Delta \hat{z}_{j}= \begin{cases}\bar{h}_{o j}-z_{j}(k), & \text { if } z_{j}(k)+\Delta \tilde{z}_{j}>\bar{h}_{o j} \\ \underline{h}_{o j}-z_{j}(k), & \text { if } z_{j}(k)+\Delta \tilde{z}_{j}<\underline{h}_{o j} \\ \Delta \tilde{z}_{j}, & \text { otherwise. }\end{cases}
$$

On the other hand, each projection subproblem in (36) has exactly the same form as the projection subproblem considered in Section III, thus can be solved by the active-set strategy based DT method.

Now we are ready to state our algorithm for solving OPF problems with large number of thermal-limit constraints stated in (17) as follows.

Algorithm III:

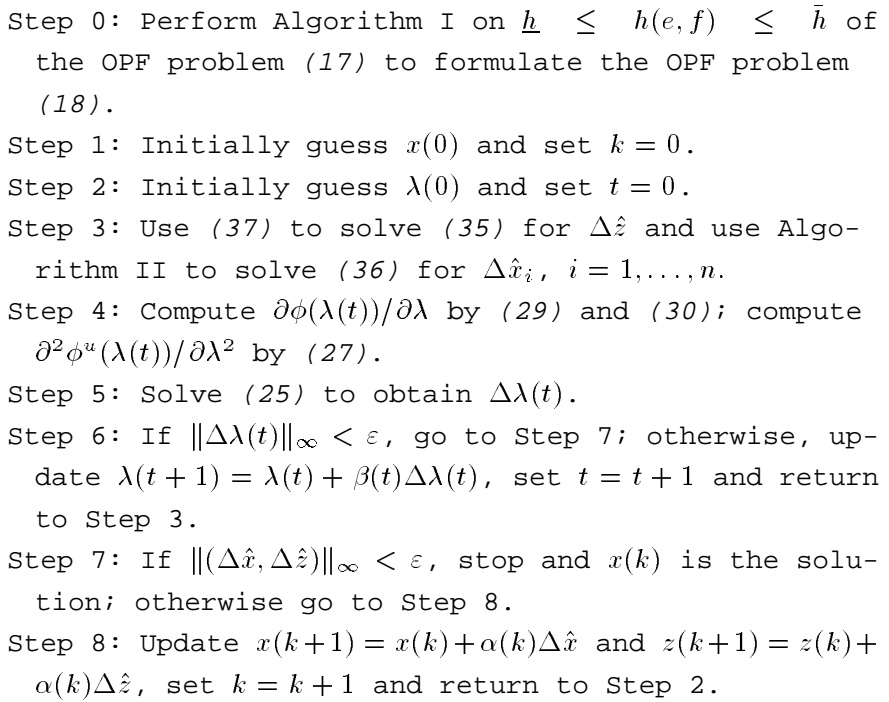

Remark 7: For the issues of discrete control variables and problem of infeasibility, we follow the same treatments as that of [11], although in current algorithm, we do not express them explicitly.

\section{Test Results}

We have applied Algorithm III on numerous OPF problems with large number of thermal-limit constraints of the IEEE 118-bus system and the IEEE 244-bus system. Due to the page limitation of the paper, we present the simulation results of the IEEE 244-bus system only while the results of the IEEE 118-bus system can be found in [15].

We set the following parameters: $L_{0}=4, T_{0}=10, C_{0}=20$ in Algorithm I, $\delta_{1}=0.01, \varepsilon=10^{-3}$ in Algorithm II, and $\delta_{2}=0.01, \epsilon=10^{-3}$ in Algorithm III. The objective functions for all the tested OPF problems are of minimum system losses criteria as $\sum_{t_{i j}} P_{t_{i j}}$, where $P_{t_{i j}}$ denotes the real power loss of transmission line $t_{i j}$, and total generation cost criteria as $\sum_{G_{i}} a_{i} P_{G_{i}}^{2}+b_{i} P_{G_{i}}^{2}+c_{i}$ where $P_{G_{i}}$ denotes the real power generation of bus $i$ and $a_{i}, b_{i}$, and $c_{i}$ are the coefficients of the cost curve of $P_{G_{i}}$. We consider four different cases of thermal-limit constraints for the IEEE 244-bus system, and the corresponding transmission lines of these four cases are a) $38-40,38-110,54-110,40-42,42-80,80-34,34-82$, $82-72,72-84,84-23,23-21,21-22,22-15,15-14,14-12$, $12-4,4-1,1-10,10-7,7-175,175-226,226-211,211-177$, 177-219, 219-203, 203-202, 202-235, 235-237, 237-238 and 238-201, b) 54-110, 54-63, 63-110, 38-110, 38-40, 40-42, $42-80,80-34,34-82,82-72,72-84,84-80,80-67,67-83$, $83-20,20-22,22-15,15-14,14-12,10-7,7-175,175-226$, 226-211, 211-177, 177-219, 219-203, 203-202, 203-235, 202-235, 235-237 and 237-238, c) 12-17, 17-14, 14-118, $118-143,12-4,4-1,1-10,10-11,11-144,144-163,163-149$, 149-164, 164-143, 10-7, 7-175, 175-226, 226-218, 218-210, 210-195, 195-227, 227-212, 212-26, 26-215, 215-217, 217-205, 205-204, 204-201, 201-109, 109-197, 197-211 and 211-226 d) 54-63, 63-110, 54-40, 38-110,38-82, 82-42, $42-80,80-34,34-82,82-72,72-74,74-86,86-84,72-84$, 84-63, 63-21, 21-22, 22-15, 15-14, 14-12, 12-4, 4-1, 1-10, 10-7, 7-175, 175-226, 226-211, 211-196, 196-197, 211-197, 197-219, 219-203, 203-235, 235-237, 237-202 and 202-235.

Applying Algorithm I, which is Step 0 of Algorithm III, to the above four cases of thermal-limit constraints, we found that the thermal-limit constraints of a), b), c), and d) cases belong to a), b), c), and d) categories considered in Section II, respectively. The removed transmission lines determined by Algorithm I are 12-4 for case a), 22-15 for case b), 17-175 and 201-109 for case c), and 15-14 for case d). For the transmission lines of each of the a), b), c), and d) cases, we assume four sets of thermal-limit values to account for different number of active thermal-limit constraints. Now, we have formed 32 OPF problems of the form (18) on the IEEE 244-bus system, and each OPF problem is resulted from a combination of the two objective function criteria, four cases of thermal-limit constraints and four different number of active thermal-limit constraints. We then apply the rest of the steps of Algorithm III to these 32 OPF problems in a Sparc 20 machine. The corresponding CPU times are shown in Tables I-VIII. Each table consists of four OPFs corresponding to four cases of thermal-limit constraints with a given criteria and a given percentage of active thermal-limit constraints. For the purpose of comparison, we also solve the same OPF problems with the same setup by the SQP method associated with DT method using pure conversion technique in the same Sparc 20 machine. This method is given in [11] and is abbreviated as SQPDTCON in the sequel. The final objective values obtained by SQPDTCON method are the same as the values obtained by Algorithm III, and the corresponding CPU times consumption for each OPF are also reported in Tables I-VIII. The test results shown in Table I and Table V correspond to the case that no thermal-limit constraints is active, thus the resulting CPU times of Algorithm III are the least compared with the other cases of same criteria but with active thermal limit constraints. Similar results also hold for the SQPDTCON method. Tables II-IV report the CPU times consumed by the Algorithm III and SQPDTCON method for the cases of the OPF problems of minimum system-losses criteria but with approximately $7 \%, 13 \%$, and $20 \%$ active thermallimit constraints. As we can see from the fifth column of these three tables that the speed-up ratio of Algorithm III over the 
TABLE I

COMPARISON OF THE ALGORITHM III AND THE SQPDTCON METHOD ON OPF PRoBlEMS OF THE IEEE 244-BUS SySTEMS WITH MINIMUM SYSTEM-LOSSES CRITERIA AND NO ACTIVE THERMAL-LIMIT CONSTRAINT

\begin{tabular}{|c|c|c|c|c|c|}
\hline Methods & $\begin{array}{l}\text { Algorithm } \\
\text { III }\end{array}$ & $\begin{array}{l}\text { SQPDT- } \\
\text { CON }\end{array}$ & SQPDT & \multirow{2}{*}{$\begin{array}{l}\text { Speed- } \\
\text { up ratio } \\
\frac{(i i)}{(i)}\end{array}$} & \multirow{2}{*}{$\begin{array}{l}\text { CPU } \\
\text { times in- } \\
\text { creased } \\
\frac{(i)-(i i i)}{(i i i)}\end{array}$} \\
\hline Cases & $\begin{array}{l}\text { CPU } \\
\text { times } \\
\text { (sec.) } \\
\text { (j) }\end{array}$ & $\begin{array}{l}\text { CPU } \\
\text { times } \\
\text { (sec.) } \\
\text { (ii) }\end{array}$ & $\begin{array}{l}\text { CPU } \\
\text { times } \\
\text { (sec.) } \\
\text { (iii) }\end{array}$ & & \\
\hline (a) & 0.650 & 7.377 & \multirow{4}{*}{0.605} & 2.03 & $7.44 \%$ \\
\hline (b) & 0.671 & 7.414 & & 2.11 & $10.91 \%$ \\
\hline (c) & 0.712 & 1.687 & & 2.37 & $77.69 \%$ \\
\hline (d) & 0.673 & 1.489 & & 2.21 & $17.24 \%$ \\
\hline
\end{tabular}

TABLE II

COMPARISON OF THE ALGORITHM III AND THE SQPDTCON METHOD ON OPF PROBLEMS OF THE IEEE 244-BUS SYSTEMS WITH MINIMUM SYSTEM-LOSSES CRITERIA AND 7\% ACTIVE THERMAL-LIMIT CONSTRAINTS

\begin{tabular}{|c|c|c|c|c|c|}
\hline Methods & $\begin{array}{l}\text { Algorithm } \\
\text { III }\end{array}$ & $\begin{array}{l}\text { SQPDT- } \\
\text { CON }\end{array}$ & SQPDT & \multirow{2}{*}{$\begin{array}{l}\text { Speed- } \\
\text { up ratio } \\
\frac{(i i)}{(i)}\end{array}$} & \multirow{2}{*}{$\begin{array}{l}\text { CPU } \\
\text { times in- } \\
\text { creased } \\
\frac{(i)-(i i i)}{(i i i)}\end{array}$} \\
\hline Cases & $\begin{array}{l}\text { CPU } \\
\text { times } \\
\text { (sec.) } \\
\text { (i) }\end{array}$ & $\begin{array}{l}\text { CPU } \\
\text { times } \\
\text { (sec.) } \\
\text { (ii) }\end{array}$ & $\begin{array}{l}\text { CPU } \\
\text { times } \\
\text { (sec.) } \\
\text { (iii) }\end{array}$ & & \\
\hline (a) & 0.695 & 1.408 & \multirow{4}{*}{0.605} & 2.03 & $14.88 \%$ \\
\hline (b) & 0.716 & 1.504 & & 2.10 & $18.18 \%$ \\
\hline (c) & 0.757 & 1.780 & & 2.35 & $25.12 \%$ \\
\hline (d) & 0.717 & 1.578 & & 2.20 & $18.51 \%$ \\
\hline
\end{tabular}

TABLE III

COMPARISON OF THE ALGORITHM III AND THE SQPDTCON METHOD ON OPF PROBLEMS OF THE IEEE 244-BUS SYSTEMS WITH MINIMUM SYSTEM-LOSSES CRITERIA AND 13\% ACTIVE THERMAL-LIMIT CONSTRAINTS

\begin{tabular}{|c|c|c|c|c|c|}
\hline Methods & $\begin{array}{l}\text { Algorithm } \\
\text { III }\end{array}$ & $\begin{array}{l}\text { SQPDT- } \\
\text { CON }\end{array}$ & SQPDT & \multirow{2}{*}{$\begin{array}{l}\text { Speed- } \\
\text { up ratio } \\
\frac{(i i)}{(i)}\end{array}$} & \multirow{2}{*}{$\begin{array}{l}\text { CPU } \\
\text { times in- } \\
\text { creased } \\
\frac{(i)-(i i i)}{(i i i)}\end{array}$} \\
\hline Cases & $\begin{array}{l}\text { CPU } \\
\text { times } \\
\text { (sec.) } \\
\text { (i) }\end{array}$ & $\begin{array}{l}\text { CPU } \\
\text { times } \\
\text { (sec.) } \\
\text { (ii) }\end{array}$ & $\begin{array}{l}\text { CPU } \\
\text { times } \\
\text { (sec.) } \\
\text { (iii) }\end{array}$ & & \\
\hline (a) & 0.741 & 1.498 & \multirow{4}{*}{0.605} & 2.02 & $22.48 \%$ \\
\hline (b) & 0.760 & 1.595 & & 2.10 & $25.61 \%$ \\
\hline (c) & 0.803 & 1.871 & & 2.33 & $32.72 \%$ \\
\hline (d) & 0.764 & 1.670 & & 2.19 & $26.28 \%$ \\
\hline
\end{tabular}

TABLE IV

COMPARISON OF THE ALGORITHM III AND THE SQPDTCON METHOD ON OPF PROBLEMS OF THE IEEE 244-BUS SYSTEMS WITH MINIMUM SYSTEM-LOSSES CRITERIA AND 20\% ACTIVE THERMAL-LIMIT CONSTRAINTS

\begin{tabular}{|c|c|c|c|c|c|}
\hline Methods & $\begin{array}{l}\text { Algorithm } \\
\text { III }\end{array}$ & $\begin{array}{l}\text { SQPDT- } \\
\text { CON }\end{array}$ & SQPDT & \multirow{2}{*}{$\begin{array}{l}\text { Speed- } \\
\text { up ratio } \\
\frac{(i i)}{(i)}\end{array}$} & \multirow{2}{*}{$\begin{array}{l}\text { CPU } \\
\text { times in } \\
\text { creased } \\
\frac{(i)-(i a i)}{(i q i)}\end{array}$} \\
\hline Cases & $\begin{array}{l}\text { CPU } \\
\text { times } \\
\text { (sec.) } \\
\text { (i) }\end{array}$ & $\begin{array}{l}\text { CPU } \\
\text { times } \\
\text { (sec.) } \\
\text { (ii) }\end{array}$ & $\begin{array}{l}\text { CPU } \\
\text { times } \\
\text { (sec.) } \\
\text { (iii) }\end{array}$ & & \\
\hline (a) & 0.786 & 1.590 & \multirow{4}{*}{0.605} & 2.02 & $29.91 \%$ \\
\hline (b) & 0.806 & 1.688 & & 2.09 & $33.22 \%$ \\
\hline (c) & 0.845 & 1.963 & & 2.32 & $39.67 \%$ \\
\hline (d) & 0.809 & 1.761 & & 2.18 & $33.72 \%$ \\
\hline
\end{tabular}

SQPDTCON method is about 2. This indicates the improvement of the current method on the SQPDTCON method is more than $100 \%$. The reason that the CPU time of Algorithm III and its speed-up ratio over the SQPDTCON method are approximately the same for the four cases of thermal-limit constraints in a table is because the disjoint subgraphs have approximately the same capacities and the number of removed transmission
TABLE V

COMPARISON OF THE ALGORITHM III AND THE SQPDTCON METHOD ON OPF PROBLEMS OF THE IEEE 244-BUS SySTEMS WITH TOTAL GENERATION COST CRITERIA AND NO ACTIVE THERMAL-LIMIT CONSTRAINT

\begin{tabular}{|c|c|c|c|c|c|}
\hline Methods & $\begin{array}{l}\text { Algorithm } \\
\text { III }\end{array}$ & $\begin{array}{l}\text { SQPDT- } \\
\text { CON }\end{array}$ & SQPDT & \multirow{2}{*}{$\begin{array}{l}\text { Speed- } \\
\text { up ratio } \\
\frac{(i i)}{(i)}\end{array}$} & \multirow{2}{*}{$\begin{array}{l}\text { CPU } \\
\text { times in- } \\
\text { creased } \\
\frac{(i)-(i i i)}{(i i i)}\end{array}$} \\
\hline Cases & $\begin{array}{l}\text { CPU } \\
\text { times } \\
\text { (sec.) } \\
\text { (i) }\end{array}$ & $\begin{array}{l}\text { CPU } \\
\text { times } \\
\text { (sec.) } \\
\text { (ii) }\end{array}$ & $\begin{array}{l}\text { CPU } \\
\text { times } \\
\text { (sec.) } \\
\text { (iii) }\end{array}$ & & \\
\hline (a) & 0.674 & 1.417 & \multirow{4}{*}{0.612} & 2.10 & $10.13 \%$ \\
\hline (b) & 0.678 & 1.518 & & 2.24 & $10.78 \%$ \\
\hline (c) & 0.710 & 1.548 & & 2.18 & $16.01 \%$ \\
\hline (d) & 0.690 & 1.593 & & 2.31 & $72.75 \%$ \\
\hline
\end{tabular}

TABLE VI

COMPARISON OF THE ALGORITHM III AND THE SQPDTCON METHOD ON OPF PROBLEMS OF THE IEEE 244-Bus SySTEMS WITH TOTAL GENERATION COST CRITERIA AND 7\% ACTIVE THERMAL-LIMIT CONSTRAINTS

\begin{tabular}{|c|c|c|c|c|c|}
\hline Methods & $\begin{array}{l}\text { Algorithm } \\
\text { III }\end{array}$ & $\begin{array}{l}\text { SQPDT- } \\
\text { CON }\end{array}$ & SQPDT & \multirow{2}{*}{$\begin{array}{l}\text { Speed- } \\
\text { up ratio } \\
\frac{(i i)}{(i)}\end{array}$} & \multirow{2}{*}{$\begin{array}{l}\text { CPU } \\
\text { times in- } \\
\text { creased } \\
\frac{(i)-(i i i)}{(i i i)}\end{array}$} \\
\hline Cases & $\begin{array}{l}\text { CPU } \\
\text { times } \\
\text { (sec.) } \\
\text { (i) }\end{array}$ & $\begin{array}{l}\text { CPU } \\
\text { times } \\
\text { (sec.) } \\
\text { (ii) }\end{array}$ & $\begin{array}{l}\text { CPU } \\
\text { times } \\
\text { (sec.) } \\
\text { (iii) }\end{array}$ & & \\
\hline (a) & 0.719 & 1.508 & \multirow{4}{*}{0.612} & 2.10 & $17.48 \%$ \\
\hline (b) & 0.723 & 1.607 & & 2.22 & $18.14 \%$ \\
\hline (c) & 0.754 & 1.640 & & 2.12 & $23.20 \%$ \\
\hline (d) & 0.737 & 1.684 & & 2.28 & $20.42 \%$ \\
\hline
\end{tabular}

TABLE VII

COMPARISON OF THE ALGORITHM III AND THE SQPDTCON METHOD ON OPF PROBLEMS OF THE IEEE 244-BUS SYSTEMS WITH TOTAL GENERATION COST CRITERIA AND 13\% ACTIVE THERMAL-LIMIT CONSTRAINTS

\begin{tabular}{|c|c|c|c|c|c|}
\hline Methods & $\begin{array}{l}\text { Algorithm } \\
\text { III }\end{array}$ & $\begin{array}{l}\text { SQPDT- } \\
\text { CON }\end{array}$ & SQPDT & \multirow{2}{*}{$\begin{array}{l}\text { Speed- } \\
\text { up ratio } \\
\frac{(i i)}{(i)}\end{array}$} & \multirow{2}{*}{$\begin{array}{l}\text { CPU } \\
\text { times in } \\
\text { creased } \\
\frac{(i)-(i i i)}{(i i i)}\end{array}$} \\
\hline Cases & $\begin{array}{l}\text { CPU } \\
\text { times } \\
\text { (sec.) } \\
\text { (i) }\end{array}$ & $\begin{array}{l}\text { CPU } \\
\text { times } \\
\text { (sec.) } \\
\text { (ii) }\end{array}$ & $\begin{array}{l}\text { CPU } \\
\text { times } \\
\text { (sec.) } \\
\text { (iii) }\end{array}$ & & \\
\hline (a) & 0.761 & 7.597 & \multirow{4}{*}{0.612} & 2.10 & $24.35 \%$ \\
\hline (b) & 0.768 & 1.699 & & 2.21 & $25.49 \%$ \\
\hline (c) & 0.798 & 1.732 & & 2.17 & $30.39 \%$ \\
\hline (d) & 0.781 & 1.775 & & 2.27 & $27.61 \%$ \\
\hline
\end{tabular}

TABLE VIII

COMPARISON OF THE ALGORITHM III AND THE SQPDTCON METHOD ON OPF PROBLEMS OF THE IEEE 244-BUS SySTEMS WITH TOTAL GENERATION COST CRITERIA AND 20\% ACTIVE THERMAL-LIMIT CONSTRAINTS

\begin{tabular}{|c|c|c|c|c|c|}
\hline Methods & $\begin{array}{l}\text { Algorithm } \\
\text { III }\end{array}$ & $\begin{array}{l}\text { SQPDT- } \\
\text { CON }\end{array}$ & SQPDT & \multirow{2}{*}{$\begin{array}{l}\text { Speed- } \\
\text { up ratic } \\
\frac{(i i)}{(i)}\end{array}$} & \multirow[b]{2}{*}{$\begin{array}{l}\text { CPU } \\
\text { times in } \\
\text { creased } \\
\frac{(i)-(i i i)}{(\text { iii })}\end{array}$} \\
\hline Cases & $\begin{array}{l}\text { CPU } \\
\text { times } \\
\text { (sec.) } \\
\text { (i) } \\
\end{array}$ & $\begin{array}{l}\text { CPU } \\
\text { times } \\
\text { (sec.) } \\
\text { (ii) }\end{array}$ & $\begin{array}{l}\text { CPU } \\
\text { times } \\
\text { (sec.) } \\
\text { (iii) }\end{array}$ & & \\
\hline (a) & 0.806 & 1.688 & \multirow{4}{*}{0.612} & 2.09 & $32.70 \%$ \\
\hline (b) & 0.811 & 1.788 & & 2.20 & $32.52 \%$ \\
\hline (c) & 0.844 & 1.823 & & 2.16 & $37.91 \%$ \\
\hline (d) & 0.827 & 1.866 & & 2.26 & $35.13 \%$ \\
\hline
\end{tabular}

lines are almost the same. However, the CPU times consumed by Algorithm III increase when there are more active thermal-limit constraints present, which are as we expected and can be observed from Tables I-IV. Similar results can also be observed from Tables V-VIII for the OPF problems with total generation cost criteria. 
To investigate the efficiency of the two new techniques for handling the large number of thermal-limit constraints, we also solve the same OPF problem but without considering any thermal-limit constraint using the SQP method associated with the DT method. We abbreviate this method as SQPDT method which in fact is the SQPDTCON method without using conversion technique, because no thermal-limit constraint is considered. Thus, the SQPDT method solves only two OPF problems, one for each criteria. For easier reference, we also show the CPU times consumption of the SQPDT method in tables with the same objective function. We also report the increased CPU times of Algorithm III compared with the SQPDT method in each table, and they are mostly around $10 \%$ when no thermal-limit constraint is active and less than $40 \%$ when there are $20 \%$ active thermal-limit constraints. These results indicate that the two new techniques are very efficient in handling the large number of thermal-limit constraints.

Remark 8: Unfortunately, we do not have any software implemented by the interior point method at hand to compare with. However, in [11], we did compare the SQPDTCON method with IMSL subroutine and achieve a speed-up ratio of 30 on a modified 57-bus system.

\section{CONCLUSION}

In this paper, we have proposed two new techniques to improve the previously developed DT method [11] used in solving OPF problems with large number of thermal-limit constraints. These two new techniques can decompose the large-dimension projection problem into independent medium-dimension projection subproblems and solve the medium-dimension projection subproblems very efficiently; their computational efficiency and effective improvement on the DT method have been demonstrated by the results of our tests on numerous OPF problems.

The DT method [11] is not only developed for the OPF problems, it can also apply to the nonlinear multicommodity network flow problems [16]. Therefore, the two new techniques proposed in this paper possess the potential to generalize the DT method in [11] and [16] to solve general large network optimization problems.

\section{REFERENCES}

[1] B. Stott, J. L. Marinho, and O. Alsac, "Review of linear programming applied to power system rescheduling PICA," PICA, pp. 142-154, 1979.
[2] B. Stott and J. L. Marinho, "Linear programming for power-system network security applications," IEEE Trans. Power Apparat. Syst., vol. PAS-98, pp. 837-848, May 1979.

[3] B. Scott, O. Alsac, and A. Monticelli, "Security analysis and optimization," Proc. IEEE, vol. 75, no. 12, pp. 1623-1664, Dec. 1987.

[4] T. C. Giras and S. N. Talukdar, "Quasi-Newton method for optimal power flows," Int. J. Elect. Power Energy Syst., vol. 3, no. 2, pp. 59-64, Apr. 1981.

[5] S. N. Talukdar and T. C. Giars, "A fast and robust variable metric method for optimum power flows," IEEE Trans. Power Apparat. Syst., vol. PAS-101, pp. 415-420, Feb. 1982.

[6] R. C. Burchett, H. H. Happ, and D. R. Vierath, "Quadratically convergent optimal power flow," IEEE Trans. Power Apparat. Syst., vol. PAS-103, pp. 3267-3275, Nov. 1985.

[7] D. I. Sun, B. Ashley, B. Brewer, A. Hughes, and W. F. Tinney, "Optimal power flow by Newton approach,” IEEE Trans. Power Apparat. Syst., vol. PAS-103, pp. 2864-2880, Oct. 1984.

[8] D. I. Sun, T. I. Hu, G. S. Lin, C. J. Lin, and C. M. Chen, "Experiences with implementing optimal power flow for reactive scheduling in the Taiwan power system," IEEE Trans. Power Syst., vol. 3, pp. 1193-1120, Aug. 1988.

[9] A. Monticelli and W. E. Liu, "Adaptive movement penalty method for the Newton optimal power flow," IEEE Trans. Power Syst., vol. 7, pp. 334-340, Feb. 1992.

[10] Y. C. Wu, A. S. Debs, and R. E. Marsten, "A direct nonlinear predictorcorrector primal-dual interior point algorithm for optimal power flows," IEEE Trans. Power Syst., vol. 9, pp. 876-883, May 1994.

[11] C. -H. Lin and S. -Y. Lin, "A new dual-type method used in solving optimal power flow problems," IEEE Trans. Power Syst., vol. 12, pp. 1667-1675, Nov. 1997

[12] A. S. Debs, Modern Power Systems Control and Operation. Boston, MA: Kluwer, 1988.

[13] R. Gould, Graph Theory. Menlo Park, CA: Benjamin/Cummings, 1988.

[14] D. Luenberger, Linear and Nonlinear Programming, 2nd ed. Reading, MA: Addison-Wesley, 1984.

[15] C. H. Lin, "A new dual-type method for optimal power flow problems," Kao Yuan Institute of Technology, Dept. Elect. Eng., Kaoshiung, Taiwan, R.O.C., TR-2001, 2001.

[16] S. Y. Lin and C. H. Lin, "A computational efficient method for nonlinear mutlticommodity network flow problems," Networks, vol. 29, pp. 225-244, July 1997.

Ch'i-Hsin Lin is currently an Assistant Professor in the Electronics Engineering Department at KaoYuan Institute of Technology, Kaoshiung, Taiwan, R.O.C.

Shin-Yeu Lin is currently a Professor in the Department of Electrical and Control Engineering at National Chiao Tung University, Hsinchu, Taiwan, R.O.C.

Shieh-Shing Lin is currently pursuing the Ph.D degree in the Department of Electrical and Control Engineering at National Chiao Tung University, Hsinchu, Taiwan, R.O.C. 